\title{
Bit Error Rate Analysis of Rectangular QAM/FSO Systems Using an APD Receiver Over Atmospheric Turbulence Channels
}

\author{
Bach T. Vu, Ngoc T. Dang, Truong C. Thang, and Anh T. Pham
}

\begin{abstract}
We theoretically analyze the performance of free-space optical (FSO) systems using rectangular quadrature-amplitude modulation (QAM) and an avalanche photodiode (APD) receiver over atmospheric turbulence channels. Both log-normal and gamma-gamma channel models are used in the analysis for the cases of weak/moderate and strong atmospheric turbulence. The system bit error rate, when Gray code mapping is employed, is theoretically derived taking into account various link conditions and system parameters, including the APD shot noise, thermal noise, channel attenuation and geometrical loss, atmospheric turbulence strengths, and link distances. The numerical results show that using APD with a proper selection of the average gain could greatly benefit the performance of the system; as a matter of fact, in the case of optimal gain, the system using an APD receiver could provide $7 \mathrm{~dB}$ gain in comparison with the one with a positive-instrinsic-negative receiver. We also quantitatively discuss the impact of link conditions and system parameters on the selection of optimal APD gain.
\end{abstract}

Index Terms-Atmospheric turbulence; Avalanche photodiode (APD); Free-space optics (FSO); Quadratureamplitude modulation (QAM).

\section{INTRODUCTION}

I $\mathrm{n}$ recent years, free-space optical (FSO) communication has received much attention, from both industry and academia, as an alternative solution for terrestrial broadband wireless access over short distances [티, $]$. This is thanks to its advantages of cost-effectiveness, quick and easy deployment, and high data-rate provision. Especially when the radio-frequency ( $\mathrm{RF}$ ) spectrum is heavily congested, the feature of license-free service becomes a significant advantage of FSO communications.

In terrestrial FSO communication, the primary factors that degrade system performance are atmospheric attenuation and turbulence. Atmospheric attenuation, which is caused by absorption and scattering processes, is variable and difficult to predict, and hence significantly limits the

Manuscript received October 9, 2012; revised February 18, 2013; accepted March 8, 2013; published April 15, 2013 (Doc. ID 177560).

The authors are with the Computer Communications Laboratory, University of Aizu, Fukushima 965-8580, Japan (email: pham@u-aizu.ac.jp).

http://dx.doi.org/10.1364/JOCN.5.000437 coverage of FSO systems. Atmospheric turbulence is a phenomenon occurring when there are variations in the refractive index due to inhomogeneity in temperature and pressure changes [3]. This index inhomogeneity could deteriorate the quality of the received signal and lead to an increase in the bit error rate (BER) of the FSO systems.

Conventionally, FSO systems have mainly been implemented by employing on-off keying (OOK) modulation because of the simplicity and low cost. In OOK modulation, the correct selection of adaptive thresholds is critical to the performance. However, due to the fluctuation of the signal intensity, adaptive threshold adjustment is difficult to accomplish. Also, an alternative modulation technique, pulse-position modulation (PPM), has been proposed for FSO communication [4]. It has been found that PPM has superior power efficiency compared to OOK; however, it has poor bandwidth efficiency.

To overcome the limitations of OOK and PPM, subcarrier phase-shift keying (PSK) has been proposed and extensively studied [5-8]. Furthermore, in order to achieve a better spectral efficiency than that of PSK, the use of subcarrier quadrature-amplitude modulation (QAM) for FSO systems has been recently proposed. The average symbol error rate (ASER) of FSO systems using subcarrier intensity-modulated rectangular QAM over log-normal and gamma-gamma turbulence channels was derived in [9]. In [10], Hassan et al. derived the closed-form expression of ASER for gamma-gamma and $K$-distributed and negative exponential channels using a series expansion of the modified Bessel function. In these studies (even the electrical signal-to-noise ratio (SNR) was defined as a function of background irradiance and thermal noise in [10]), investigation of ASER versus SNR over atmospheric turbulence channels has been carried out without considering the impact of receiver noise. Additionally, the impact of link loss, including both atmospheric attenuation and geometric loss, has been ignored in all studies.

In this paper, we therefore present a theoretical study on the performance of FSO systems employing subcarrier intensity-modulated rectangular QAM. In particular, instead of the ASER as in previous studies, we theoretically derive the system BER, a better indication of system performance, when Gray code mapping is employed. In the 
BER derivation, we take into account various atmospheric link conditions, including distance, turbulence strength, atmospheric attenuation, and geometrical loss. Both lognormal and gamma-gamma fading channels are used in the analysis for the cases of weak/moderate and strong atmospheric turbulence.

Additionally, in the BER analysis, we also consider the impact of the receiver noise, including signal shot noise and thermal noise. In particular, we theoretically analyze and compare the performance of the systems using an avalanche photodiode (APD) receiver with that of the ones using a positive-instrinsic-negative ( $p-i-n)$ receiver. It is worth noting that the APD receiver has been proposed for FSO systems in several previous studies. In [4] Kiasaleh investigated the performance of APD-based FSO systems using PPM signaling. Cvijetic et al. analyzed the impact of the APD receiver on the performance of FSO systems using PPM with spatial diversity [11,12]. The use of APD arrays for the cases of FSO systems using binary PPM and OOK modulations was reported in [13]. There has been, however, no study on the impact of an APD receiver on the performance of rectangular QAM/FSO systems. In the numerical analysis, we therefore obtain important performance bounds for the practical system design, such as the effects of APD gain, transmitted power, receiver temperature, and link distance on the BER of rectangular QAM/FSO systems. In particular, the gain selection in different systems and link conditions will also be discussed.

The remainder of the paper is organized as follows. The rectangular QAM/FSO system using an APD receiver is presented in Section II. Section III describes the channel model considering the total link loss and atmospheric turbulence. In Section IV, the system BER is theoretically derived. Numerical results are presented in Section V, and finally, Section VI presents the conclusions of the study.

\section{System ModeL}

A typical FSO system using subcarrier intensitymodulated rectangular QAM and an APD receiver is depicted in Fig. 1. At the transmitter side, each block of $\log _{2} M$ data bits is first modulated by an electrical $\left(M_{I} \times M_{Q}\right)$ QAM modulator, where $M_{I}$ and $M_{Q}$ are, respectively, the dimensions of the in-phase and quadrature

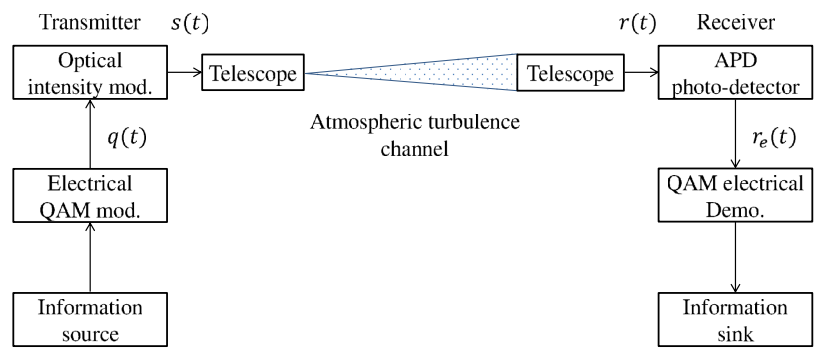

Fig. 1. System diagram of subcarrier intensity-modulated rectangular QAM/FSO system over an atmospheric turbulence channel. signals, and $M=M_{I} \times M_{Q}$. The signal at the output of the electrical QAM modulator can be expressed as

$$
q(t)=s_{I}(t) \cos \left(2 \pi f_{c} t\right)-s_{Q}(t) \sin \left(2 \pi f_{c} t\right),
$$

where $f_{c}$ is the QAM subcarrier frequency and $s_{I}(t)=$ $\sum_{j=-\infty}^{\infty} a_{j} g\left(t-j T_{s}\right)$ and $s_{Q}(t)=\sum_{j=-\infty}^{\infty} b_{j} g\left(t-j T_{s}\right)$ are the in-phase and quadrature signals, respectively. $a_{j}$ and $b_{j}$ are the in-phase and quadrature components of the jth amplitude symbol, respectively. $g(t)$ is the shaping pulse, and $T_{s}$ denotes the symbol interval.

The QAM signal is used to modulate the intensity of a laser of the transmitter, and the transmitted signal can be written as

$$
s(t)=P_{s}\left\{1+m\left[s_{I}(t) \cos \left(2 \pi f_{c} t\right)-s_{Q}(t) \sin \left(2 \pi f_{c} t\right)\right]\right\},
$$

where $P_{s}$ is the transmitted optical power and $m$ is the modulation index. Due to the effect of link loss and atmospheric turbulence, the received signal can be expressed as

$$
r(t)=a_{v} X(t) s(t)
$$

In this equation, $a_{v}$ represents the link loss and $X(t)$ is the random process for the signal scintillation caused by atmospheric turbulence. These two parameters will be discussed in more detail in Section III. Also at the receiver side, a telescope narrows the laser beam and projects it toward the APD receiver. From Eqs. (2) and (3), the received optical intensity at the input of the APD receiver can be written as

$$
r(t)=a_{v} X(t) P_{s}\left\{1+m\left[s_{I}(t) \cos \left(2 \pi f_{c} t\right)-s_{Q}(t) \sin \left(2 \pi f_{c} t\right)\right]\right\} .
$$

As the dc term $P_{s} a_{v} X(t)$ can be filtered out by a bandpass filter, denoting $\Re$ and $\bar{g}$ as the APD's responsivity and average gain, respectively, the electrical signal at the output of the APD can be expressed as

$$
\begin{aligned}
r_{e}(t)= & \bar{g} \Re a_{v} X(t) P_{s}\left\{m \left[s_{I}(t) \cos \left(2 \pi f_{c} t\right)\right.\right. \\
& \left.\left.-s_{Q}(t) \sin \left(2 \pi f_{c} t\right)\right]\right\}+n(t)
\end{aligned}
$$

Here, $n(t)$ is the total receiver noise, consisting of APD shot noise, thermal noise, and dark current. For the sake of simplicity, we assume that the dark current noise is negligible; $n(t)$ therefore can be given by

$$
n(t)=i_{\mathrm{Sh}}(t)+i_{\mathrm{Th}}(t)
$$

where $i_{\mathrm{Sh}}(t)$ and $i_{\mathrm{Th}}(t)$ are the APD shot noise and thermal noise, respectively. The thermal noise is modeled as stationary zero-mean Gaussian noise with the variance $\sigma_{\text {Th }}^{2}$ given as

$$
\sigma_{\mathrm{Th}}^{2}=\left(4 k_{B} \frac{T}{R_{L}}\right) F_{n} \Delta f,
$$

in which $k_{B}, T, R_{L}, F_{n}$, and $\Delta f$ denote the Boltzmann constant, the receiver's temperature in degrees Kelvin, the 
APD's load resistance, the amplifier noise figure, and the symbol's effective noise bandwidth, respectively [14].

The effect of scintillation on APD shot noise leads to the uncertainty in shot noise variance. The atmospheric turbulence link, however, can be modeled as a slow-fading channel, because its temporal correlation time, which is on the order of several milliseconds [15], is much larger than the QAM symbol duration. It is thus possible to consider samples of the turbulence process at a given time $x=X\left(t=t_{0}\right)$. As a result, the APD shot noise there can be treated as zeromean Gaussian noise with variance $\sigma_{\mathrm{Sh}}^{2}$ being conditioned on $x$ as

$$
\sigma_{\mathrm{Sh}}^{2}=2 q \bar{g}^{2} F_{A} \Re a_{v} x P_{s} m \Delta f,
$$

where $q$ denotes electron charge and $F_{A}$ is the excess noise factor of the APD and is given by $F_{A}=k_{A} \bar{g}+$ $\left(1-k_{A}\right)(2-1 / \bar{g})$, with $k_{A}$ denoting the ionization factor [14]. The total variance of APD noise therefore can be expressed as

$$
\sigma_{n}^{2}=2 q \bar{g}^{2} F_{A} \Re a_{v} x P_{s} m \Delta f+\left(4 k_{B} \frac{T}{R_{L}}\right) F_{n} \Delta f .
$$

The instantaneous electrical SNR per symbol, denoted as $\gamma$ at the output of the APD, is therefore expressed as a function of instantaneous fading value $x$, with system and channel parameters as follows:

$$
\gamma=\frac{\left(\bar{g} \Re a_{v} x P_{s}\right)^{2}}{2 q \bar{g}^{2} F_{A} \Re a_{v} x P_{s} m \Delta f+\left(4 k_{B} \frac{T}{R_{L}}\right) F_{n} \Delta f} .
$$

\section{Channel Model}

During the propagation over the FSO channel, the optical signal's amplitude and phase are distorted by various atmospheric interferences, from scattering, refraction, and absorption to rainfall, fog, and dust. In our study, we consider three primary factors characterizing an FSO channel including atmospheric attenuation, geometric loss, and atmospheric turbulence. For the atmospheric turbulence, both log-normal and gamma-gamma channel models will be considered for the cases of weak/moderate and strong atmospheric turbulence.

\section{A. Link Loss}

The link loss consists of atmospheric turbulence and geometric loss. The atmospheric attenuation is caused by both molecular absorption and aerosol scattering suspended in the air, while the geometric loss is determined by the angle of divergence of the transmitter and the area of the receiver's aperture. Mathematically, the total link loss can be expressed as

$$
a_{v}=\frac{A}{\pi\left(\frac{\Theta L}{2}\right)^{2}} e^{\left(-\beta_{v} L\right)},
$$

where $\beta_{v}, L, A$, and $\Theta$ are the atmospheric extinction coefficient, the link distance between the transmitter and the receiver, the area of the optical receiver, and the angle of divergence in radians, respectively [3].

\section{B. Turbulence Models}

When the optical signal propagates through an FSO channel, scintillation $X(t)$ is characterized by a stationary random process. In the log-normal turbulence channel, assuming that the average of scintillation is normalized to unity, the probability density function (pdf) $f_{X}(x)$ is given by

$$
f_{X}(x)=\frac{1}{x \sigma_{S} \sqrt{2 \pi}} \exp \frac{-\left[\ln x+\frac{\sigma_{S}^{2}}{2}\right]^{2}}{2 \sigma_{S}^{2}},
$$

where $\sigma_{S}^{2}$ is the scintillation index, which depends on the channel's characteristics. It is given by Eq. (10) of [9] as

$$
\begin{aligned}
\sigma_{S}^{2}= & \exp \left[\frac{0.49 \sigma_{2}^{2}}{\left(1+0.18 d^{2}+0.56 \sigma_{2}^{12 / 5}\right)^{7 / 6}}\right. \\
& \left.+\frac{0.51 \sigma_{2}^{2}\left(1+0.69 \sigma_{2}^{12 / 5}\right)^{-5 / 6}}{\left(1+0.90 d^{2}+0.62 d^{2} \sigma_{2}^{12 / 5}\right)}\right]-1,
\end{aligned}
$$

in which $d$ depends on the link distance $L$ and the aperture diameter of receiver $D$ as $d=\sqrt{k D^{2} / 4 L}$ and $k=2 \pi / \lambda$ is the optical wave number. The Rytov variance in the case of spherical wave propagation, $\sigma_{2}^{2}$, can be expressed as

$$
\sigma_{2}^{2}=0.492 C_{n}^{2} k^{7 / 6} L^{11 / 6},
$$

where $C_{n}^{2}$ is the strength of the turbulence. For a horizontal path of up to a few kilometers, the value of $C_{n}^{2}$ is taken to be a constant.

When the turbulence becomes stronger, however, the lognormal distribution generates a significant difference from the experimental results [16]. The reason is that the log-normal pdf underestimates the behavior of the tails as compared with measurement results. In this case, the gamma-gamma channel model is used, and its normalized pdf is given as

$$
f_{X}(x)=\frac{2(\alpha \beta)^{(\alpha+\beta) / 2}}{\Gamma(\alpha) \Gamma(\beta)} x^{(\alpha+\beta) / 2-1} K_{\alpha-\beta}(2 \sqrt{\alpha \beta x}),
$$

where $K_{i}(\cdot)$ denotes the modified Bessel function of the second kind and order $i . \Gamma(\cdot)$ denotes the gamma function [3]. For spherical wave propagation, the parameters $\alpha$ and $\beta$ can be expressed in terms of Rytov variance, defined above, as 


$$
\begin{gathered}
\alpha=\left\{\exp \left[\frac{0.49 \sigma_{2}^{2}}{\left(1+0.18 d^{2}+0.56 \sigma_{2}^{12 / 5}\right)^{7 / 6}}\right]-1\right\}^{-1}, \\
\beta=\left\{\exp \left[\frac{0.51 \sigma_{2}^{2}\left(1+0.69 \sigma_{2}^{12 / 5}\right)^{-5 / 6}}{\left(1+0.9 d^{2}+0.62 d^{2} \sigma_{2}^{12 / 5}\right)}\right]-1\right\}^{-1} .
\end{gathered}
$$

\section{BER ANALYSIS}

In this section, we will analyze the performance of the QAM/FSO systems using an APD receiver. As the atmospheric turbulence can be modeled as a slow-fading channel, the system BER therefore can be derived as a general expression as follows:

$$
\operatorname{BER}=\int_{0}^{\infty} \operatorname{BER}_{\text {inst }} f_{X}(x) \mathrm{d} x
$$

To derive the BER, we first consider a specific case of the popular $8 \times 4 \mathrm{QAM}$ to illustrate the analysis, then we provide the expression of the generalized BER for an arbitrary rectangular $M$-QAM case.

\section{A. BER for an $8 \times 4 Q A M$ Scheme}

As mentioned in the previous section, the 32-QAM signal constellation scheme can be considered as the combination of two independent 8-PAM ("PAM" denotes pulseamplitude modulation) and 4-PAM signals with $M_{I}=8$ and $M_{Q}=4$, respectively. The signal-space diagram of the $8 \times 4$ QAM is shown in Fig. 2, in which the in-phase and quadrature decision distances are $2 d_{I}$ and $2 d_{Q}$, respectively. Each $8 \times 4$ QAM symbol $\left(x_{1}, x_{2}, x_{3}, x_{4}, x_{5}\right)$ includes two components, an 8-PAM symbol $\left(x_{1}, x_{3}, x_{5}\right)$ and a 4-PAM symbol $\left(x_{2}, x_{4}\right)$. In the analysis, a perfect Gray code is also assumed and all symbols are transmitted with equal probability. An individual bit error in a $8 \times 4$ QAM symbol can exclusively result from a bit error in either 8-PAM or 4-PAM. The average instantaneous BER for an $8 \times 4$ QAM can be calculated from the average bit error in the two PAM signals and can be expressed as

$$
\mathrm{BER}_{\text {inst }}=\frac{1}{\log _{2}(8 \times 4)}\left(\sum_{k=1}^{\log _{2} 8} P_{8}(k)+\sum_{l=1}^{\log _{2} 4} P_{4}(l)\right),
$$

where $P_{8}(k)$ and $P_{4}(l)$ are the probability of bit error happening at the $k$ th or $l$ th bit in the 8-PAM signals on the in-phase channel or 4-PAM signals on the quadrature channel, respectively.

1) I-Channel Error: When the gray code is used for mapping signals into symbols, the effect of the noise will lead to the different error probability on each bit. Considering the first bit $i_{1}$, the error will occur if the noise exceeds $\pm 7 d_{I}$, $\pm 5 d_{I}, \pm 3 d_{I}, \pm d_{I}$ for $111,011,110,010,100,000,101$, 001 symbols, respectively. Moreover, each symbol was transmitted with a probability of $1 / 8$; thus, the error rate on the first bit is given by

$$
\begin{aligned}
P_{8}(1)= & \frac{1}{8}\left[\operatorname{erfc}\left(\frac{d_{I}}{\sqrt{2 \sigma_{n}^{2}}}\right)+\operatorname{erfc}\left(\frac{3 d_{I}}{\sqrt{2 \sigma_{n}^{2}}}\right)\right. \\
& \left.+\operatorname{erfc}\left(\frac{5 d_{I}}{\sqrt{2 \sigma_{n}^{2}}}\right)+\operatorname{erfc}\left(\frac{7 d_{I}}{\sqrt{2 \sigma_{n}^{2}}}\right)\right] .
\end{aligned}
$$

For the second bit $i_{2}$, considering the transmitted symbol 111 , error happens when the noise exceeds $3 d_{I}$ but is still less than $11 d_{I}$. Applying the same analysis yields the BER expression for the second bit $P_{8}(2)$ and for the third bit $P_{8}(3)$ as follows:

$$
\begin{aligned}
P_{8}(2)= & \frac{1}{8}\left[2 \cdot \operatorname{erfc}\left(\frac{d_{I}}{\sqrt{2 \sigma_{n}^{2}}}\right)+2 \cdot \operatorname{erfc}\left(\frac{3 d_{I}}{\sqrt{2 \sigma_{n}^{2}}}\right)+\operatorname{erfc}\left(\frac{5 d_{I}}{\sqrt{2 \sigma_{n}^{2}}}\right)\right. \\
& \left.+\operatorname{erfc}\left(\frac{7 d_{I}}{\sqrt{2 \sigma_{n}^{2}}}\right)-\operatorname{erfc}\left(\frac{9 d_{I}}{\sqrt{2 \sigma_{n}^{2}}}\right)-\operatorname{erfc}\left(\frac{11 d_{I}}{\sqrt{2 \sigma_{n}^{2}}}\right)\right], \quad(21)
\end{aligned}
$$

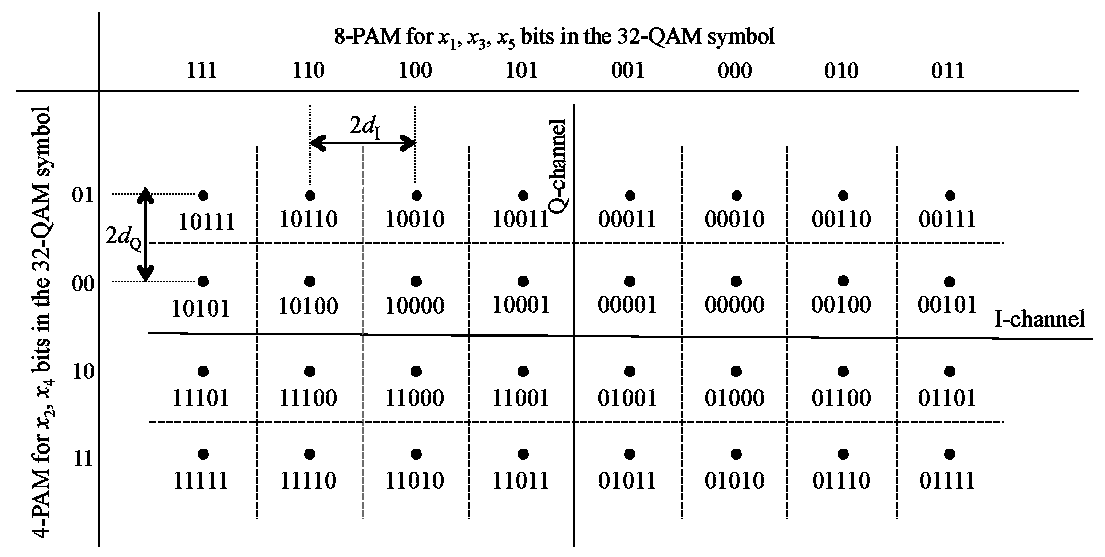

Fig. 2. Signal-space diagram for the $8 \times 4$ QAM scheme. 


$$
\begin{aligned}
P_{8}(3)= & \frac{1}{8}\left[4 \cdot \operatorname{erfc}\left(\frac{d_{I}}{\sqrt{2 \sigma_{n}^{2}}}\right)+3 \cdot \operatorname{erfc}\left(\frac{3 d_{I}}{\sqrt{2 \sigma_{n}^{2}}}\right)-3 \operatorname{erfc}\left(\frac{5 d_{I}}{\sqrt{2 \sigma_{n}^{2}}}\right)\right. \\
& +2 \operatorname{erfc}\left(\frac{7 d_{I}}{\sqrt{2 \sigma_{n}^{2}}}\right)-2 \operatorname{erfc}\left(\frac{9 d_{I}}{\sqrt{2 \sigma_{n}^{2}}}\right) \\
& \left.+\operatorname{erfc}\left(\frac{11 d_{I}}{\sqrt{2 \sigma_{n}^{2}}}\right)-\operatorname{erfc}\left(\frac{13 d_{I}}{\sqrt{2 \sigma_{n}^{2}}}\right)\right]
\end{aligned}
$$

2) Q-Channel Error: The analysis of instantaneous BER analysis for 4-PAM can also be obtained by applying the above method, and it can be expressed as

$$
\begin{gathered}
P_{4}(1)=\frac{1}{4}\left[\operatorname{erfc}\left(\frac{d_{Q}}{\sqrt{2 \sigma_{n}^{2}}}\right)+\operatorname{erfc}\left(\frac{3 d_{Q}}{\sqrt{2 \sigma_{n}^{2}}}\right)\right], \\
P_{4}(2)=\frac{1}{4}\left[2 \cdot \operatorname{erfc}\left(\frac{d_{Q}}{\sqrt{2 \sigma_{n}^{2}}}\right)+\operatorname{erfc}\left(\frac{3 d_{Q}}{\sqrt{2 \sigma_{n}^{2}}}\right)-\operatorname{erfc}\left(\frac{5 d_{Q}}{\sqrt{2 \sigma_{n}^{2}}}\right)\right] .
\end{gathered}
$$

Finally, we obtained the instantaneous BER for an $8 \times 4$ QAM by substituting Eqs. (20)-(24) into Eq. (19).

\section{B. BER for the General $M_{I} \times M_{Q} Q A M$ Scheme}

For the $M_{I} \times M_{Q}$ rectangular QAM, the system BER can be obtained by generalizing 8-PAM and 4-PAM in Eq. (19). As the result of that, the instantaneous BER is written as

$$
\mathrm{BER}_{\mathrm{inst}}=\frac{1}{\log _{2}\left(M_{I} M_{Q}\right)}\left(\sum_{k=1}^{\log _{2} M_{I}} P_{M_{I}}(k)+\sum_{l=1}^{\log _{2} M_{Q}} P_{M_{Q}}(l)\right),
$$

where $P_{M_{I}}(k)$ and $P_{M_{Q}}(l)$ denote the BER occurring on the $k$ th bit of $M_{I}$-ary PAM and the $l$ th bit of $M_{Q}$-ary PAM, respectively. Regularly, the BER of $M_{I}$-PAM and $M_{Q}$-PAM schemes can be evaluated via the Euclidean distance between two adjacent symbols, $2 d_{I}$ and $2 d_{Q}$, respectively, as follows:

$$
\begin{gathered}
\frac{d_{I}}{\sqrt{2 \sigma_{n}^{2}}}=\sqrt{\frac{3 \gamma}{2\left(\left(M_{I}^{2}-1\right)+\zeta^{2}\left(M_{Q}^{2}-1\right)\right)}}, \\
\frac{d_{Q}}{\sqrt{2 \sigma_{n}^{2}}}=\sqrt{\frac{3 \zeta^{2} \gamma}{2\left(\left(M_{I}^{2}-1\right)+\zeta^{2}\left(M_{Q}^{2}-1\right)\right)}} .
\end{gathered}
$$

In the above equations, $\gamma$ is the instantaneous electrical SNR per symbol given in Eq. (10) and $\zeta=d_{Q} / d_{I}$ is the quadrature-to-inphase decision distance ratio. Using the derivation of the probability that the $k$ th bit in error for the $I$-ary PAM scheme in [17], $P_{M_{I}}(k)$ and $P_{M_{Q}}(l)$ can be respectively given by

$$
\begin{aligned}
P_{M_{I}}(k)= & \frac{1}{M_{I}} \sum_{i=0}^{\left(1-2^{-k}\right) M_{I}-1}\left\{(-1)^{\left\lfloor\frac{i \cdot 2^{k-1}}{M_{I}}\right\rfloor}\left(2^{k-1}-\left\lfloor\frac{i \cdot 2^{k-1}}{M_{I}}+\frac{1}{2}\right\rfloor\right)\right. \\
& \left.\times \operatorname{erfc}\left((2 i+1) \frac{d_{I}}{\sqrt{2 \sigma_{n}^{2}}}\right)\right\}, \\
P_{M_{Q}}(l)= & \frac{1}{M_{Q}} \sum_{j=0}^{\left(1-2^{-l}\right) M_{Q}-1}\left\{(-1)^{\left.\frac{j \cdot 2^{l-1}}{M_{Q}}\right\rfloor}\left(2^{l-1}-\left\lfloor\frac{j \cdot 2^{l-1}}{M_{Q}}+\frac{1}{2}\right\rfloor\right)\right. \\
& \left.\times \operatorname{erfc}\left((2 i+1) \frac{d_{Q}}{\sqrt{2 \sigma_{n}^{2}}}\right)\right\} .
\end{aligned}
$$

Here, $\lfloor z\rfloor$ is the largest integer to $z$.

Substituting Eqs. (26) and (27) into Eqs. (28) and (29), $P_{M_{I}}(k)$ and $P_{M_{Q}}(l)$ can be written as

$$
\begin{aligned}
P_{M_{I}}(k)= & \frac{1}{M_{I}} \sum_{i=0}^{\left(1-2^{-k}\right) M_{I}-1}\left\{(-1)^{\left\lfloor\frac{i \cdot 2^{k-1}}{M_{I}}\right\rfloor}\left(2^{k-1}-\left\lfloor\frac{i \cdot 2^{k-1}}{M_{I}}+\frac{1}{2}\right\rfloor\right)\right. \\
& \left.\times \operatorname{erfc}\left((2 i+1) \sqrt{\frac{3 \gamma}{2\left(\left(M_{I}^{2}-1\right)+\zeta^{2}\left(M_{Q}^{2}-1\right)\right)}}\right)\right\},
\end{aligned}
$$

$$
\begin{aligned}
P_{M_{Q}}(l)= & \frac{1}{M_{Q}} \sum_{j=0}^{\left(1-2^{-l}\right) M_{Q}-1}\left\{(-1)^{\left.\frac{\mid \cdot 2^{2} M_{Q}-1}{M_{Q}}\right\rfloor}\left(2^{l-1}-\left\lfloor\frac{j \cdot 2^{l-1}}{M_{Q}}+\frac{1}{2}\right\rfloor\right)\right. \\
& \left.\times \operatorname{erfc}\left((2 j+1) \sqrt{\frac{3 \zeta^{2} \gamma}{2\left(\left(M_{I}^{2}-1\right)+\zeta^{2}\left(M_{Q}^{2}-1\right)\right)}}\right)\right\} .
\end{aligned}
$$

Replacing Eqs. (30) and (31) with the instantaneous electrical SNR $\gamma$ defined in Eq. (10) into Eq. (25), we obtain the expression of the instantaneous BER for rectangular QAM/FSO systems using an APD receiver. The BER is then calculated by using Eq. (18) with $f_{X}(x)$ for the log-normal and gamma-gamma channels given in Eqs. (12) and (15), respectively. Defining $P_{I}$ and $P_{Q}$ as

$$
\begin{gathered}
P_{I}=\sum_{k=1}^{\log _{2} M_{I}} \int_{0}^{\infty} P_{M_{I}}(k) f_{X}(x) \mathrm{d} x, \\
P_{Q}=\sum_{l=1}^{\log _{2} M_{Q}} \int_{0}^{\infty} P_{M_{Q}}(l) f_{X}(x) \mathrm{d} x,
\end{gathered}
$$

the system BER can be written as

$$
\mathrm{BER}=\frac{1}{\log _{2}\left(M_{I} M_{Q}\right)}\left(P_{I}+P_{Q}\right) .
$$

\section{Analytical Expression of the BER}

In this section, we develop the analytical expression for the system BER in the case of log-normal and 
gamma-gamma channels. For this purpose, analytical expressions of $P_{I}$ and $P_{Q}$ given in Eqs. (32) and (33), respectively, will be derived. In Eq. (하), denoting $C_{i} \frac{1}{M_{I}}(-1)^{\left\lfloor\cdot \cdot^{k-1}\right\rfloor}\left(2^{k-1}-\left\lfloor\frac{i \cdot 2^{k-1}}{M_{I}}+\frac{1}{2}\right\rfloor\right) \quad$ and $\quad A_{i}=(2 i+1)$ $\sqrt{\frac{3}{2\left(\left(M_{I}^{2}-1\right)+\zeta^{2}\left(M_{Q}^{2}-1\right)\right)}}, P_{M_{I}}(k)$ and $P_{M_{Q}}(l)$ can be rewritten as

$$
\begin{aligned}
& P_{M_{I}}(k)=\sum_{i=0}^{\left(1-2^{-k}\right) M_{I}-1} C_{i} \operatorname{erfc}\left(A_{i} \sqrt{\gamma}\right), \\
& P_{M_{Q}}(l)=\sum_{j=0}^{\left(1-2^{-l}\right) M_{Q^{-1}}} C_{j} \operatorname{erfc}\left(A_{j} \sqrt{\gamma}\right),
\end{aligned}
$$

with $\quad C_{j} \frac{1}{M_{Q}}(-1)^{\left\lfloor\cdot \frac{j \cdot 2_{Q}-1}{M_{Q}}\right\rfloor}\left(2^{l-1}-\left\lfloor\frac{j \cdot 2^{l-1}}{M_{Q}}+\frac{1}{2}\right\rfloor\right) \quad$ and $\quad A_{j}=$ $(2 j+1) \sqrt{\frac{3}{2\left(\left(M_{I}^{2}-1\right)+\zeta^{2}\left(M_{Q}^{2}-1\right)\right)}}$.

To facilitate our calculation, let $a=\left(\bar{g} \Re a_{v} P_{s}\right)^{2}$, $b=2 q \bar{g}^{2} F_{A} \Re a_{v} P_{s} m \Delta f$, and $c=\left(4 k_{B} \frac{T}{R_{L}}\right) F_{n} \Delta f$; thus, the instantaneous electrical SNR $\gamma$ in Eq. $\underline{\text { (10) }}$ is given by

$$
\gamma=\frac{a x^{2}}{b x+c}
$$

\section{A. Log-Normal Channel}

By transforming the random variable $y=\frac{\left(\ln x+\sigma_{S}^{2} / 2\right)}{\sqrt{2} \sigma_{S}}, P_{I}$ can be expressed as

$$
\begin{aligned}
P_{I}= & \sum_{k=1}^{\log _{2} M_{I}} \sum_{i=0}^{\left(1-2^{-k}\right) M_{I}-1} C_{i} \frac{1}{\sqrt{\pi}} \\
& \times \int_{-\infty}^{\infty} \exp \left(-y^{2}\right) \operatorname{erfc}\left(A_{i} \sqrt{\frac{a \exp \left(2 \sqrt{2} \sigma_{S} y-\sigma_{S}^{2}\right)}{b \exp \left(\sqrt{2} \sigma_{S} y-\sigma_{S}^{2} / 2\right)+c}}\right) \mathrm{d} y
\end{aligned}
$$

Using the Gauss-Hermite quadrature integration, the approximation of $P_{I}$ and $P_{Q}$ can be expressed, respectively, as

$$
\begin{aligned}
P_{I} \approx & \sum_{k=1}^{\log _{2} M_{I}} \sum_{i=0}^{\left(1-2^{-k}\right) M_{I}-1} \sum_{t=-N ; t \neq 0}^{N} C_{i} \frac{1}{\sqrt{\pi}} \\
& \times w_{t} \operatorname{erfc}\left(A_{i} \sqrt{\left.\frac{a \exp \left(2 \sqrt{2} \sigma_{S} y_{t}-\sigma_{S}^{2}\right)}{b \exp \left(\sqrt{2} \sigma_{S} y_{t}-\sigma_{S}^{2} / 2\right)+c}\right)},\right. \\
P_{Q} \approx & \sum_{l=1}^{\log _{2} M_{Q}\left(1-2^{-l}\right) M_{Q^{-1}}} \sum_{j=0}^{N} C_{t=-N ; t \neq 0} \frac{1}{\sqrt{\pi}} \\
& \times w_{t} \operatorname{erfc}\left(A_{j} \sqrt{\frac{a \exp \left(2 \sqrt{2} \sigma_{S} y_{t}-\sigma_{S}^{2}\right)}{b \exp \left(\sqrt{2} \sigma_{S} y_{t}-\sigma_{S}^{2} / 2\right)+c}}\right),
\end{aligned}
$$

where $w_{t}$ and $y_{t}$ with $t=(-N,-N+1, \ldots, 1,2, \ldots, N)$ denote the weight factors and the zeros of the Hermite polynomial, respectively.

\section{B. Gamma-Gamma Channel}

Also using the generalized power series representation of a modified Bessel function of the second kind as in Eq. (6) of [18], we first express $f_{X}(x)$ as

$$
f_{X}(x)=\sum_{n=0}^{\infty}\left(a_{n}(\alpha, \beta) x^{n+\beta-1}+a_{n}(\beta, \alpha) x^{n+\alpha-1}\right),
$$

where $(\alpha-\beta) \notin Z,|x|<\infty$ and

$$
a_{n}(\alpha, \beta)=\frac{\pi(\alpha \beta)^{n+\beta}}{\sin [\pi(\alpha-\beta)] \Gamma(\alpha) \Gamma(\beta) \Gamma(n-\alpha+\beta+1) n !} .
$$

It is notable that Eq. (41) is valid for $\alpha-\beta \notin \mathbb{Z}$ and this condition is not a severe restriction for typical values of parameters $\alpha$ and $\beta$. Making use of a simple and tight exponential bound for the $\operatorname{erfc}(x)$ function

$$
\operatorname{erfc}\left(A_{i} \sqrt{\gamma}\right) \approx \frac{1}{6} e^{-A_{i}^{2} \gamma}+\frac{1}{2} e^{-\frac{4}{3} A_{i}^{2} \gamma}
$$

we then obtain an expression for $P_{M_{I}}(k)$ as follows:

$$
P_{M_{I}}(k) \approx \sum_{i=0}^{\left(1-2^{-k}\right) M_{I}-1} C_{i}\left(\frac{1}{6} e^{-A_{i}^{2} \gamma}+\frac{1}{2} e^{-\frac{4}{3} A_{i}^{2} \gamma}\right) .
$$

By transforming random variable $\gamma=a x^{2} /(b x+c)$ in Eq. $(\underline{41}), f_{\gamma}(\gamma)$ is given by

$$
\begin{aligned}
f_{\gamma}(\gamma)= & \sum_{n=0}^{\infty} \sum_{m=0}^{\infty}\left(D(\alpha, \beta) g\left(\frac{n+\beta-1-m}{2}, \frac{n+\beta-1+m}{2}\right)\right. \\
& \left.+D(\beta, \alpha) g\left(\frac{n+\alpha-1-m}{2}, \frac{n+\alpha-1+m}{2}\right)\right) \\
& \times\left(\frac{b}{2 a}+\frac{b^{2}}{4 a \sqrt{a c}} g\left(-\frac{1}{2}, \frac{1}{2}\right)+\frac{c}{\sqrt{4 a c}} g\left(-\frac{1}{2},-\frac{1}{2}\right)\right),
\end{aligned}
$$

where

$$
D(\alpha, \beta)=a_{n}(\alpha, \beta) \frac{1}{(2 a)^{n+\beta-1}}\left(\begin{array}{c}
n+\beta-1 \\
m
\end{array}\right) b^{m}(4 a c)^{\frac{n+\beta-1-m}{2}}
$$

and the function $g\left(z_{1}, z_{2}\right)$ is defined as

$$
g\left(z_{1}, z_{2}\right)=\left[\gamma \frac{b^{2}}{4 a c}+1\right]^{z_{1}} \gamma^{z_{2}}
$$

Combining Eqs. (32), (44), and (45) we obtain the final expression for $P_{I}$ as 


$$
\begin{aligned}
& P_{I} \approx \frac{1}{6} \sum_{k=1}^{\log _{2} M_{I}} \sum_{i=0}^{\left(1-2^{-k}\right) M_{I}-1} C_{i} \sum_{n=0}^{\infty} \sum_{m=0}^{\infty} \\
& \times\left(\frac{b}{2 a} D(\alpha, \beta) h\left(A_{i}^{2},-\frac{n+\beta-1-m}{2}, \frac{n+\beta+1+m}{2}\right)\right. \\
& +\frac{b}{2 a} D(\beta, \alpha) h\left(A_{i}^{2},-\frac{n+\alpha-1-m}{2}, \frac{n+\alpha+1+m}{2}\right) \\
& +\frac{b^{2}}{4 a \sqrt{a c}} D(\alpha, \beta) h\left(A_{i}^{2},-\frac{n+\beta-2-m}{2}, \frac{n+\beta+2+m}{2}\right) \\
& +\frac{b^{2}}{4 a \sqrt{a c}} D(\beta, \alpha) h\left(A_{i}^{2},-\frac{n+\alpha-2-m}{2}, \frac{n+\alpha+2+m}{2}\right) \\
& +\frac{c}{\sqrt{4 a c}} D(\alpha, \beta) h\left(A_{i}^{2},-\frac{n+\beta-2-m}{2}, \frac{n+\beta+m}{2}\right) \\
& \left.+\frac{c}{\sqrt{4 a c}} D(\beta, \alpha) h\left(A_{i}^{2},-\frac{n+\alpha-2-m}{2}, \frac{n+\alpha+m}{2}\right)\right) \\
& +\frac{1}{2} \sum_{k=1}^{\log _{2} M_{I}} \sum_{i=0}^{\left(1-2^{-k}\right) M_{I}-1} C_{i} \sum_{n=0}^{\infty} \sum_{m=0}^{\infty} \\
& \times\left(\frac{b}{2 a} D(\alpha, \beta) h\left(\frac{4 A_{i}^{2}}{3},-\frac{n+\beta-1-m}{2}, \frac{n+\beta+1+m}{2}\right)\right. \\
& +\frac{b}{2 a} D(\beta, \alpha) h\left(\frac{4 A_{i}^{2}}{3},-\frac{n+\alpha-1-m}{2}, \frac{n+\alpha+1+m}{2}\right) \\
& +\frac{b^{2}}{4 a \sqrt{a c}} D(\alpha, \beta) h\left(\frac{4 A_{i}^{2}}{3},-\frac{n+\beta-2-m}{2}, \frac{n+\beta+2+m}{2}\right) \\
& +\frac{b^{2}}{4 a \sqrt{a c}} D(\beta, \alpha) h\left(\frac{4 A_{i}^{2}}{3},-\frac{n+\alpha-2-m}{2}, \frac{n+\alpha+2+m}{2}\right) \\
& +\frac{c}{\sqrt{4 a c}} D(\alpha, \beta) h\left(\frac{4 A_{i}^{2}}{3},-\frac{n+\beta-2-m}{2}, \frac{n+\beta+m}{2}\right) \\
& \left.+\frac{c}{\sqrt{4 a c}} D(\beta, \alpha) h\left(\frac{4 A_{i}^{2}}{3},-\frac{n+\alpha-2-m}{2}, \frac{n+\alpha+m}{2}\right)\right) \text {, }
\end{aligned}
$$

in which, the function $h(p, v, q)$ is defined as follows:

$$
h(p, v, q)=\int_{0}^{\infty} e^{-p \gamma} g(-v, q-1) \mathrm{d} \gamma .
$$

By applying Eq. (3.3835) in [19], the closed-form expression for $h(p, v, q)$ can be written as

$$
\begin{aligned}
h(p, v, q)= & \frac{\pi^{2}}{p^{q} \Gamma(v) \sin [\pi(q-v)]} \\
& \times\left[\left(\frac{p}{z}\right)^{v} \frac{L_{-v}^{v-q}\left(\frac{p}{z}\right)}{\sin (\pi v) \Gamma(1-q)}-\left(\frac{p}{z}\right)^{q} \frac{L_{-q}^{q-v}\left(\frac{p}{z}\right)}{\sin (\pi q) \Gamma(1-v)}\right],
\end{aligned}
$$

where $L_{v}^{q}(p)$ is a Laguerre polynomial function with $v \neq \pm 1, \pm 2, \ldots$, and $\operatorname{Re} q>0, \operatorname{Re} p>0, z=\frac{b^{2}}{4 a c}$.

In a similar way, the analytical expression of $P_{Q}$ can be obtained. To evaluate Eq. (48), a proper truncation is used. It is important to note that when the upper limit is large enough (about 40), the approximation error is negligible [18].

\section{Numerical Results}

In this section, we present numerical results for BER analysis of the QAM/FSO systems using an APD receiver over atmospheric turbulence channels. In the analysis, the log-normal model is used for the case of weak turbulence, while the gamma-gamma model is used for moderate and strong turbulence conditions. Regarding the FSO link, the atmospheric extinction coefficient $\beta=0.1 \mathrm{~dB} / \mathrm{km}$, the diameter of the receiver's aperture $D=0.08 \mathrm{~m}$, resulting in the area of the receiver's aperture $A=\pi \frac{D^{2}}{4}$, and the angle of divergence is chosen to be $5 \mathrm{mrad}$.

In our analysis, for a fair comparison, BER is evaluated as the function of the transmitted power per bit $P_{b}=\frac{P_{s}}{\log _{2}(M)}$. Unless otherwise noted, we use the system bit rate $R_{b}=2 \mathrm{~Gb} / \mathrm{s}$; correspondingly, we have the symbol effective noise bandwidth $\Delta f=0.5 \frac{R_{b}}{\log _{2}(M)}$. Also, we fix several system parameters, including the operational wavelength $\lambda=1.55 \mu \mathrm{m}$, the receiver load resistance $R_{L}=1000 \Omega$, the amplifier noise figure $F_{n}=2$, and the ionization factor $k_{A}=0.7$ for a typical InGaAs APD.

Figures 3 and 4 show the system BER versus average APD gain with different QAM schemes for weak and strong turbulence regimes, respectively. For the weak turbulence case (Fig. 3), the strength of turbulence $C_{n}^{2}=10^{-15} \mathrm{~m}^{-2 / 3}$ and the transmitted power per bit $P_{b}=-3 \mathrm{dBm}$, while for the strong turbulence case (Fig. 4), $C_{n}^{2}=3 \times$ $10^{-14} \mathrm{~m}^{-2 / 3}$ and $P_{b}=0 \mathrm{dBm}$. The link distance $L=$ $1000 \mathrm{~m}$ and three QAM schemes of $8 \times 4,8 \times 8$, and $16 \times$ 8 are considered. Clearly, the system with $8 \times 4$ offers the best performance. While the performance of the $8 \times 8$ system is a bit worse, FEC is required in the $16 \times 8$ system to achieve an acceptable BER of less than $10^{-9}$ in the case of weak turbulence. FEC is required in all systems in the strong turbulence regime, e.g., as in Fig. 4 with $C_{n}^{2}=3 \times 10^{-14} \mathrm{~m}^{-2 / 3}$.

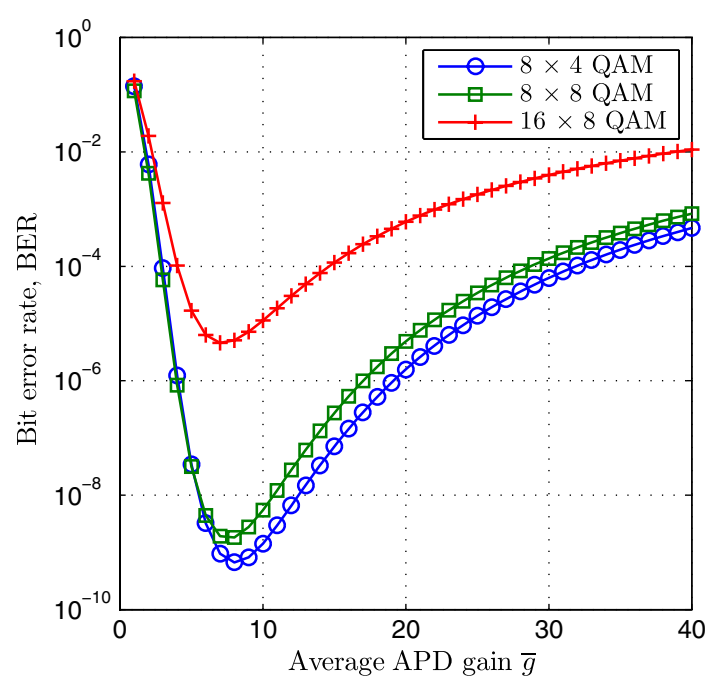

Fig. 3. BER versus average APD gain for weak turbulence and different QAM schemes, transmitted power per bit $P_{b}=-3 \mathrm{dBm}$, and $L=1000 \mathrm{~m}$. 


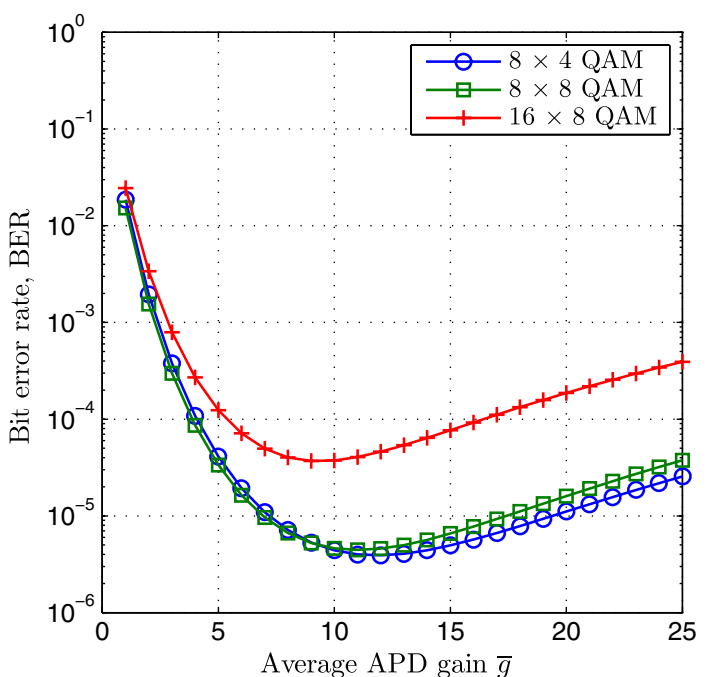

Fig. 4. BER versus average APD gain for strong turbulence and different QAM schemes, transmitted power per bit $P_{b}=0 \mathrm{dBm}$, and $L=1000 \times \mathrm{m}$.

It is also seen that the selection of APD gain has a significant impact on the system performance. In addition, the selection of optimal gain becomes more complex in the presence of stronger turbulence. For example, while the optimal gain remains almost constant in the case of weak turbulence, e.g., optimal gain is about 8 as in Fig. 3, it varies between 9 and 12 for different QAM schemes in the case of strong turbulence (Fig. 4).

In Fig. 5, we further analyze the impact of turbulence strength on the selection of optimal gain. The $8 \times 4$ QAM scheme is used. We consider three values of $C_{n}^{2}: 10^{-15}, 9 \times$ $10^{-15}$ and $3 \times 10^{-14} \mathrm{~m}^{-2 / 3}$ for the weak, moderate, and strong turbulence cases, respectively. The link distance is also $L=1000 \mathrm{~m}$ and $P_{b}=-3 \mathrm{dBm}$. Again, it is confirmed that the presence of turbulence complicates the selection of optimal gain. As is seen in Fig. 5, the

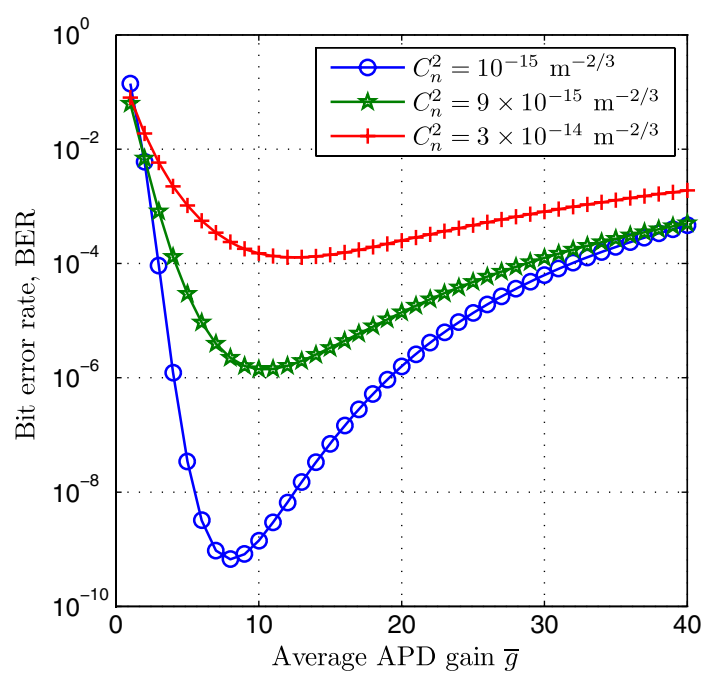

Fig. 5. BER versus average APD gain for $8 \times 4$ QAM scheme with different turbulence strengths, transmitted power per bit $P_{b}=-3 \mathrm{dBm}$, and $C_{n}^{2}=10^{-15} \mathrm{~m}^{-2 / 3}$.

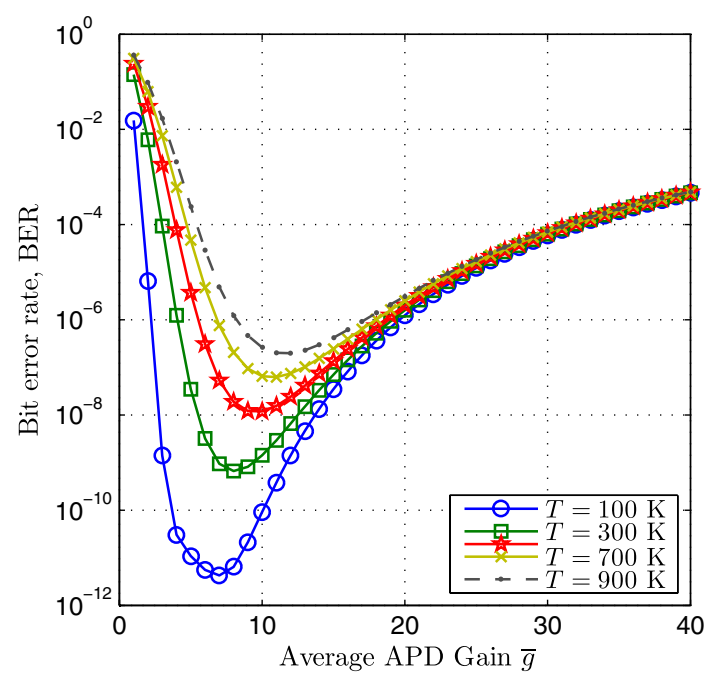

Fig. 6. BER versus average APD gain for $8 \times 4$ QAM scheme with different noise temperatures, transmitted power per bit $P_{b}=-3$ $\mathrm{dBm}$, and $C_{n}^{2}=10^{-15} \mathrm{~m}^{-2 / 3}$.

optimal APD gain increases from 8 in the case of weak turbulence to 11 when turbulence becomes stronger with $C_{n}^{2}=3 \times 10^{-14} \mathrm{~m}^{-2 / 3}$. Additionally, the impact of thermal noise on the selection of optimal gain is analyzed in Fig. 6. It is seen that the optimal gain changes more drastically when the receiver temperature varies. When the receiver temperature increases from 100 to $900 \mathrm{~K}$, it is seen that the optimal gain also varies between 8 and 12 .

Figure 7 shows the system BER versus the average transmitted power per bit $P_{b}$. We compare the performance of QAM/FSO systems when an APD and p-i-n are used with different link distances. For the APD, an optimal gain $\bar{g}=$ 10 is selected. It is seen that in both cases of link distance, the advantage of APD over p-i-n is approximately $7 \mathrm{~dB}$ at $\mathrm{BER}=10^{-8}$.

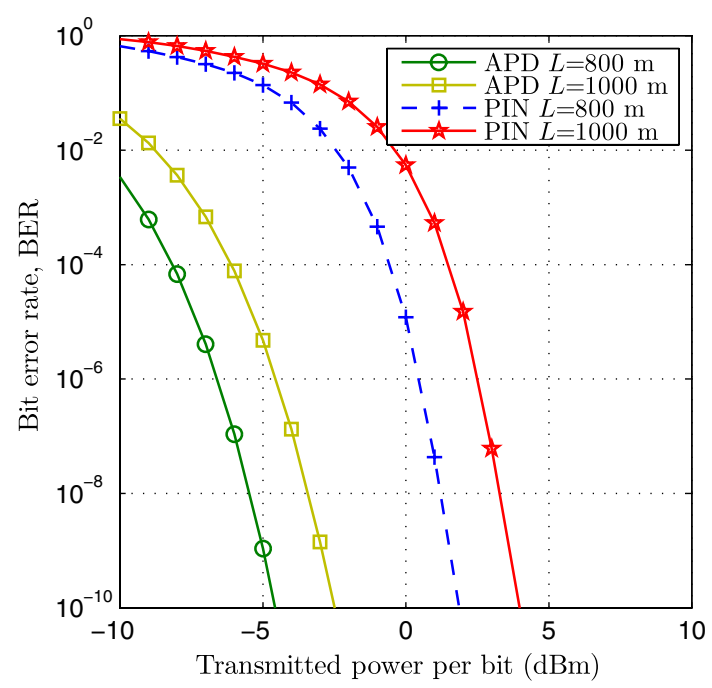

Fig. 7. BER versus average transmitted power per bit for $8 \times 4$ QAM scheme with different channel distances, $C_{n}^{2}=10^{-15} \mathrm{~m}^{-2 / 3}$, and $\bar{g}=10$. 


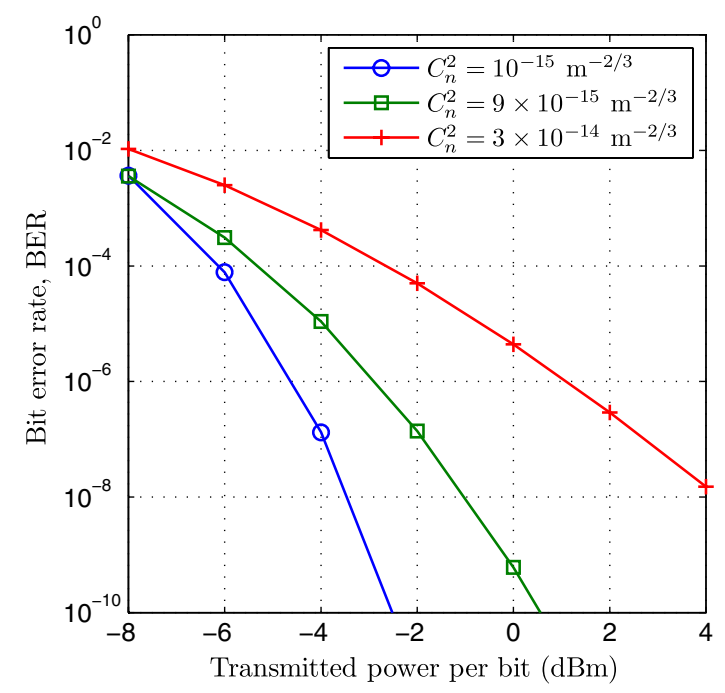

Fig. 8. BER versus average transmitted power per bit for $8 \times 4$ QAM scheme with different turbulence strengths, $L=1000 \mathrm{~m}$, and $\bar{g}=10$.

Figure 8 investigates the impact of turbulence on the transmitter power penalty. We show the BER versus transmitted power per bit $P_{b}$ for the cases of weak, moderate, and strong turbulence. With the APD gain $\bar{g}=10$ is selected, a power penalty of $2.5 \mathrm{~dB}$ is seen when the turbulence changes from weak to moderate at $\mathrm{BER}=10^{-8}$. When the turbulence is strong with $C_{n}^{2}=3 \times 10^{-14} \mathrm{~m}^{-2 / 3}$, another $5 \mathrm{~dB}$ is required to achieve similar performance.

In Fig. 9, the system BER versus the link distance with different values of average transmitted power per bit $P_{b}$, $\bar{g}=10$, and $C_{n}^{2}=10^{-15} \mathrm{~m}^{-2 / 3}$ is illustrated. This figure aims to determine the maximum distance for different transmitted power levels in the presence of turbulence. For example, when the transmitted power per bit $P_{b}=0 \mathrm{dBm}$, the link distance should not be longer than $1.5 \mathrm{~km}$ to maintain the $\mathrm{BER} \leq 10^{-8}$.

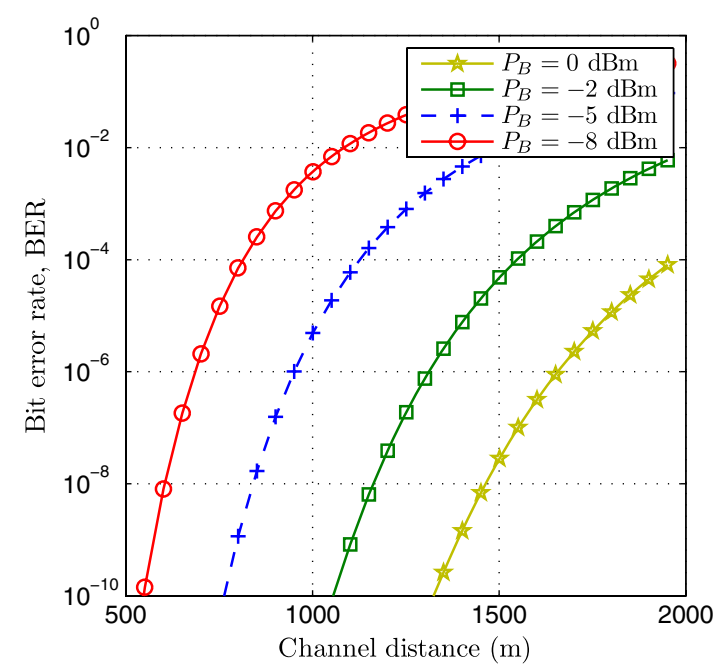

Fig. 9. BER versus channel distance for $8 \times 4$ QAM scheme and different values of average transmitted power per bit, $C_{n}^{2}=10^{-15} \mathrm{~m}^{-2 / 3}$, and $\bar{g}=10$.

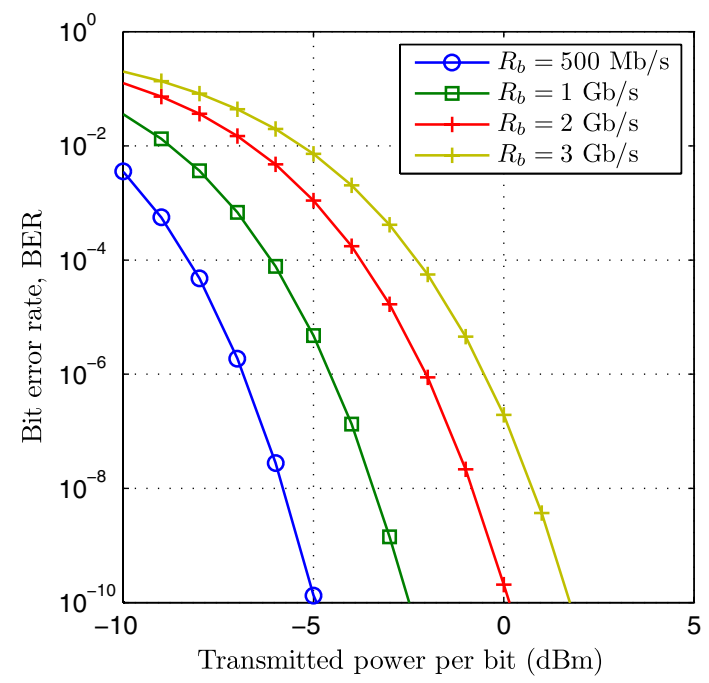

Fig. 10. BER versus transmitted power for $8 \times 4$ QAM scheme with different bit rates, $C_{n}^{2}=10^{-15} \mathrm{~m}^{-2 / 3}$, and $\bar{g}=10$.

Finally, we analyze the BER versus the average transmitted power per bit when the system bit rate varies from $500 \mathrm{Mb} / \mathrm{s}$ to $3 \mathrm{~Gb} / \mathrm{s}$ in order to determine the minimum required transmitted power for a certain bit rate. The turbulence strength $C_{n}^{2}=10^{-15} \mathrm{~m}^{-2 / 3}$ and $\bar{g}=10$. As seen in Fig. 10, for example, for the bit rate of $1 \mathrm{~Gb} / \mathrm{s}$, transmitted power per bit of $-5 \mathrm{dBm}$ is required to achieve a BER of $10^{-10}$. When the bit rate increases to $3 \mathrm{~Gb} / \mathrm{s}$, a minimum transmitted power of $-1 \mathrm{dBm}$ is required.

\section{ConcLusions}

We have theoretically analyzed the performance of FSO systems using rectangular QAM and an APD receiver over atmospheric turbulence channels. Both log-normal and gamma-gamma fading channels were used in the analysis for the cases of weak and moderate/strong atmospheric turbulence. The system BER, when Gray code mapping was employed, was theoretically derived, taking into account various system parameters and link atmospheric conditions, including the APD shot noise and thermal noise, channel attenuation and geometrical loss, atmospheric turbulence strength, and link distance.

The numerical results showed that using an APD with proper selection of the optimal gain could greatly benefit the performance of the system. Nevertheless, the thermal noise, different QAM schemes, and especially the presence of turbulence caused the selection of the optimal APD to be more sensitive. It has been shown that with different conditions of turbulence and receiver temperatures, the optimal gain could vary between 8 and 12 for a typical InGaAs APD receiver.

\section{REFERENCES}

[1] A. K. Majumdar and J. C. Ricklin, Free-Space Laser Communications: Principles and Advances. Springer, 2007. 
[2] D. O’Brien and M. Katz, "Optical wireless communications within fourth-generation wireless systems," J. Opt. Netw., vol. 4, no. 6, pp. 312-322, June 2005.

[3] S. Karp, Optical Channels: Fibers, Clouds, Water, and the Atmosphere. Plenum, 1988.

[4] K. Kiasaleh, "Performance of APD-based, PPM free-space optical communication systems in atmospheric turbulence," IEEE Trans. Commun., vol.53, no.9,pp.1455-1461, Sept. 2005.

[5] N. D. Chatzidiamantis, A. S. Lioumpas, G. K. Karagiannidis, and S. Arnon, "Adaptive subcarrier PSK intensity modulation in free space optical systems," IEEE Trans. Commun., vol. 59, no. 5, pp. 1368-1377, May 2011.

[6] Q. Lu, Q. Liu, and G. S. Mitchell, "Performance analysis for optical wireless communication systems using subcarrier PSK intensity modulation through turbulent atmospheric channel," in IEEE Global Telecommunications Conf. (GLOBECOM), 2004, vol. 3, pp. 1872-1875.

[7] Q. Liu and Q. Lu, "Subcarrier PSK intensity modulation for optical wireless communications through turbulent atmospheric channel," in IEEE Int. Conf. Communications (ICC), 2005, vol. 3, pp. 1761-1765.

[8] A. T. Pham, T. C. Thang, S. Guo, and Z. Cheng, "Performance bounds for turbo-coded SC-PSK/FSO communications over strong turbulence channels," in Int. Conf. Advanced Technologies for Communications (ATC), 2011, pp. 161-164.

[9] K. P. Peppas and C. K. Datsikas, "Average symbol error probability of general-order rectangular quadrature amplitude modulation of optical wireless communication systems over atmospheric turbulence channels," J. Opt. Commun. Netw., vol. 2, no. 2, pp. 102-110, Feb. 2010.

[10] M. Z. Hassan, X. Song, and J. Cheng, "Subcarrier intensity modulated wireless optical communications with rectangular QAM," J. Opt. Commun. Netw., vol. 4, no. 6, pp. 522-532, June 2012

[11] N. Cvijetic, S. G. Wilson, and M. Brandt-Pearce, "Receiver optimization in turbulent free-space optical MIMO channels with APDs and Q-ary PPM," IEEE Photon. Technol. Lett., vol. 19, no. 2, pp. 103-105, Jan. 2007.

[12] N. Cvijetic, S. G. Wilson, and M. Brandt-Pearce, "Performance bounds for free-space optical MIMO systems with APD receivers in atmospheric turbulence," IEEE J. Sel. Areas Commun., vol. 26, no. 3, pp. 3-12, Apr. 2008.

[13] M. Cole and K. Kiasaleh, "Receiver architectures for the detection of spatially correlated optical field using avalanche photodiode detector arrays," Opt. Eng., vol. 47, no. 2, 025008, 2008.

[14] G. P. Agrawal, Fiber-Optic Communication Systems. New York: Wiley-Interscience, 2002.

[15] J. W. Strohbehn, Laser Beam Propagations in the Atmosphere. Springer, 1978.

[16] M. A. Al-Habash, L. C. Andrews, and R. L. Phillips, "Mathematical model for the irradiance probability density function of a laser beam propagating through turbulent media," Opt. Eng., vol. 40, no. 8, pp. 1554-1562, 2001.

[17] K. Cho and D. Yoon, "On the general BER expression of one- and two-dimensional amplitude modulations," IEEE Trans. Commun., vol. 50, no. 7, pp. 1074-1080, July 2002.

[18] E. Bayaki, R. Schober, and R. K. Mallik, "Performance analysis of MIMO free-space optical systems in gamma-gamma fading," IEEE Trans. Commun., vol. 57, no. 11, pp. 3415-3424, 2009 .
[19] I. Gradshteyn and I. Ryzhik, Table of Integrals, Series, and Products. New York: Academic, 2000.

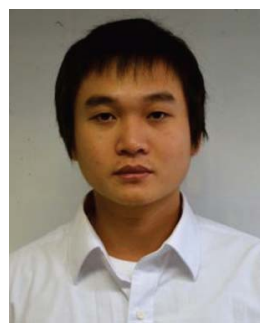

Bach T. Vu is working toward a B.E. degree in computer network systems from the University of Aizu, Japan. His study in Japan is funded by a Vietnamese government scholarship. His research interests are digital modulations, MIMO systems, and free-space optics. $\mathrm{He}$ is a student member of IEEE.

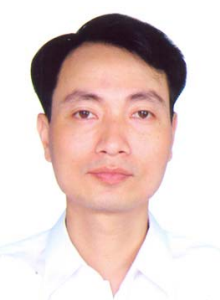

Ngoc T. Dang received a B.E. degree from Hanoi University of Technology in 1999 and an M.E. degree from Posts and Telecommunications Institute of Technology (PTIT), Vietnam, in 2005, both in electronics and telecommunications. He received a Ph.D. degree in computer science and engineering from the University of Aizu, Japan, in 2010. In 2011, he worked as an Invited Researcher at the FOTON-ENSSAT Laboratory, Universite de Rennes 1, France. In 2012, he was a JASSO Research Fellow at the Computer Communications Laboratory, University of Aizu, Japan. He is currently Deputy Head of the Department of Optical Communications at PTIT. His present research interests are in the area of optical and wireless communications.

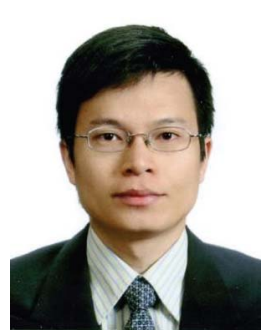

Truong C. Thang received a B.E. degree from Hanoi University of Technology, Vietnam, in 1997 and a Ph.D degree from KAIST IT Convergence Campus (KAISTICC), South Korea, in 2006. From 1997 to 2000, he worked as an Engineer at Vietnam Post and Telecom (VNPT). From 2007 to 2011, he was a Member of the Research Staff at the Electronics and Telecommunications Research Institute (ETRI), South Korea. He was also an active member of the Korean delegation to standard meetings of ISO/IEC and ITU-T from 2002 to 2011. Since 2011, he has been an Associate Professor of the University of Aizu, Japan. His research interests include multimedia networking, image/video processing, content adaptation, IPTV, and MPEG/ITU standards.

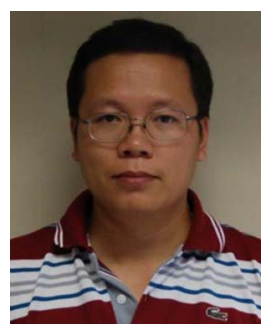

Anh T. Pham received B.E. and M.E. degrees, both in electronics engineering, from the Hanoi University of Technology, Vietnam, in 1997 and 2000, respectively, and a Ph.D. degree in information and mathematical sciences from Saitama University, Japan, in 2005. From 1998 to 2002, he was with the NTT Corp. in Vietnam. Since April 2005, he has been on the faculty at the University of Aizu, where he is currently a Senior Associate Professor at the Computer Communications Laboratory, the School of Computer Science and Engineering. His research interests are in the broad areas of communication theory and networking, with a particular emphasis on modeling, design, and performance evaluation of wired/wireless communication systems and networks. He received a Japanese government scholarship (MonbuKagaku-sho) for Ph.D. study. He also received a Vietnamese government scholarship for undergraduate study. Dr. Pham is a senior member of IEEE. He is also a member of IEICE and OSA. 